\title{
Long-term persistent symptoms of COVID-19 infection in patients with diabetes mellitus
}

\author{
Ahmed Mechi ${ }^{1} \cdot$ Alhan Al-Khalidi $^{2} \cdot$ Rasha AL-Darraji $^{3} \cdot$ Mohammed Noori Al-Dujaili $^{1} \cdot$ Karrar Al-Buthabhak $^{1}$. \\ Mohammed Alareedh ${ }^{1} \cdot$ Foaad Shaghee $^{2} \cdot$ Hussein Nafakhi $^{1}$
}

Received: 18 June 2021 / Accepted: 12 August 2021/Published online: 24 August 2021

(c) Research Society for Study of Diabetes in India 2021

\begin{abstract}
Objective The study aimed to assess the long-term persistent symptoms of patients with diabetes mellitus (DM) and COVID19 infection at 9 months after acute infection.

Methods This single-center cross-sectional study was conducted from May 20 to June 1, 2021.

Results A total of 112 patients were included in the present study. The most frequently reported persistent symptoms among DM group were fatigue $(p=0.01)$, shortness of breath $(p=0.01)$, and chest pain $(p=0.02)$ compared to non-DM group. Sulfonylurea use was associated with persistent $\operatorname{cough}(p=0.04)$.

Conclusion Long-term persistent symptoms of COVID-19 infection are common among patients with DM.
\end{abstract}

Keywords DM $\cdot$ COVID-19 $\cdot$ Long term $\cdot$ Symptoms

\section{Introduction}

Severe acute respiratory syndrome coronavirus type 2 (SARS$\mathrm{CoV}-2)$ primarily involves the respiratory tract leading to flulike symptoms and coronavirus disease-2019 (COVID-19). COVID-19 is recently recognized as a multi-organ disease often causing extra-pulmonary manifestations after the acute phase, even among those who experience mild infection [1-3].

Diabetes mellitus (DM) is major comorbidity of COVID19 infection, and patients with DM have a high prevalence of disease severity and adverse outcome during COVID-19 infection [2]. Thus, patients with COVID-19 and DM may be particularly at risk for long-term persistent symptoms or health consequences beyond the acute phase [2].

In the literature, little is known about the long-term symptoms of COVID-19 infection in patients with DM after 9 months of the acute phase. We aimed to assess the long-term persistent symptoms of patients with DM and laboratory-confirmed SARS-CoV-2 infection at 9 months after acute infection.

\section{Methods}

This is single-center cross-sectional study of patients with laboratory-confirmed SARS-CoV-2 infection who presented to the outpatient clinic of Al-Sader Teaching 
Hospital after 9 months of acute infection from May 20 to June 1, 2021.

Patients were interviewed by a trained physician in the outpatient clinic and asked to report symptoms from a pre-defined list of symptoms, including shortness of breath interfere with routine daily activities; easy fatigue; cough; chest pain; palpitation; joint pain; and neurocognitive dysfunction which includes dizziness, headache, concentration abnormalities, memory disturbances, smell loss, and taste loss. If there was a symptom that was not mentioned in the symptom questionnaire list, patients were asked to describe it. The baseline clinical comorbidities and demographic data included age, sex, hypertension, glucose-lowering drugs or insulin, smoking, body mass index (BMI), and hospital admission and duration. DM was defined as any established diagnosis prior to acute COVID-19 infection.

\section{Statistical analysis}

Statistical analysis was performed using SPSS ver. 23.0 (SPSS Inc., Chicago, IL, USA). Demographic, comorbidities, and long-term symptoms were expressed as mean \pm standard deviation for continuous variables or as numbers with percentages for categorical data. Continuous variables were compared using the Student $t$-test. The Chi-square test was used for comparisons of categorical data. $p$ Value of $<0.05$ was chosen for statistical significance.

\section{Results}

A total of 112 patients with laboratory-confirmed SARSCoV-2 at 9 months after the acute phase of COVID-19 infection were included in the present study. The patients were divided into the DM group [42 patients with age (years) $60 \pm 10,26(62 \%)$ were males] and the non-DM group [70 patients with age (years) $45 \pm 12,48(68 \%$ ) were males). The prevalence of old age, hypertension, and increased BMI was higher among the DM group than the non-DM group. Patients, characteristics are shown in Table 1.

The most frequently reported persistent symptoms among DM group compared to non-DM group were fatigue ( $76 \%$ vs. $53 \%, p=0.01)$, shortness of breath ( $45 \%$ vs. $21 \%$, $p=0.01)$, chest pain $(31 \%$ vs. $13 \%, p=0.02)$, cough $(26 \%$ vs. $13 \%, p=0.07)$, palpitation ( $21 \%$ vs. $13 \%, p=0.23)$, dizziness ( $24 \%$ vs. $10 \%, p=0.73$ ), concentration disturbances $(19 \%$ vs. $13 \%, p=0.37)$, and memory disturbances ( $17 \%$ vs. $13 \%, p=0.57)($ Table 1$)$.
Table 1 Patients' characteristics

\begin{tabular}{|c|c|c|c|}
\hline Variables & $\begin{array}{l}\mathrm{DM} \\
n=42\end{array}$ & $\begin{array}{l}\text { Non-DM } \\
n=70\end{array}$ & $p$ Value \\
\hline Age (years), mean $\pm \mathrm{SD}$ & $60 \pm 10$ & $45 \pm 12$ & $<0.01$ \\
\hline Male, $n(\%)$ & $26(62)$ & $48(68)$ & 0.55 \\
\hline $\mathrm{BMI}$, mean $\pm \mathrm{SD}$ & $32 \pm 6$ & $28 \pm 4$ & $<0.01$ \\
\hline Smoking, $n(\%)$ & $6(14)$ & $10(14)$ & 0.12 \\
\hline Hypertension, $n(\%)$ & $29(69)$ & $18(26)$ & $<0.01$ \\
\hline Hospital stay (days), mean \pm SD & $7 \pm 1$ & $5 \pm 1$ & 0.31 \\
\hline Hospital admission, $n(\%)$ & $24(57)$ & $28(40)$ & 0.07 \\
\hline \multicolumn{4}{|l|}{ Persistent symptoms } \\
\hline $\begin{array}{l}\text { At least one } \\
\text { symptom, } n(\%)\end{array}$ & $37(88)$ & $55(79)$ & 0.05 \\
\hline Fatigue, $n(\%)$ & $32(76)$ & $37(53)$ & 0.01 \\
\hline Shortness of breath, $n(\%)$ & $19(45)$ & $15(21)$ & 0.01 \\
\hline Chest pain, $n(\%)$ & $13(31)$ & $9(13)$ & 0.02 \\
\hline Cough, $n(\%)$ & $11(26)$ & $9(13)$ & 0.07 \\
\hline Joint pain, $n(\%)$ & $10(24)$ & $21(30)$ & 0.45 \\
\hline Palpitation, $n(\%)$ & $9(21)$ & $9(13)$ & 0.23 \\
\hline \multicolumn{4}{|l|}{ Neurocognitive dysfunction symptoms } \\
\hline Dizziness, $n(\%)$ & $10(24)$ & $7(10)$ & 0.73 \\
\hline Concentration abnormalities, $n(\%)$ & $8(19)$ & $9(13)$ & 0.37 \\
\hline Memory disturbances, $n(\%)$ & $7(17)$ & $9(13)$ & 0.57 \\
\hline Headache, $n(\%)$ & $6(14)$ & $14(20)$ & 0.44 \\
\hline Smell loss, $n(\%)$ & $2(5)$ & $7(10)$ & 0.48 \\
\hline Taste loss, $n(\%)$ & $1(2)$ & $5(7)$ & 0.15 \\
\hline
\end{tabular}

Regarding the relationship of diabetes lowering drugs and long-term post-COVID-19 infection symptoms, sulfonylurea use was associated with cough $(p=0.04)$ and fatigue $(p=0.05)$. Although not statistically significant, insulin use was associated with neurocognitive dysfunction, shortness of breath, and fatigue. On the other hand, metformin use was associated with decreased prevalence of shortness of breath, fatigue, and neurocognitive dysfunction compared to patients who did not use metformin, although the difference was not statistically significant (Table 2).

\section{Discussion}

We found that at 9 months after the acute COVID-19 infection, $88 \%$ of patients with DM reported at least one longterm persistent symptom, especially fatigue, neurocognitive dysfunction, and shortness of breath.

Long-term persistent musculoskeletal, respiratory, and neurocognitive symptoms have been reported for other coronaviruses (SARS and MERS) in long-term follow-up studies. Persistent viremia, relapse or reinfection, immune dysfunction, deconditioning, and post-traumatic stress have 


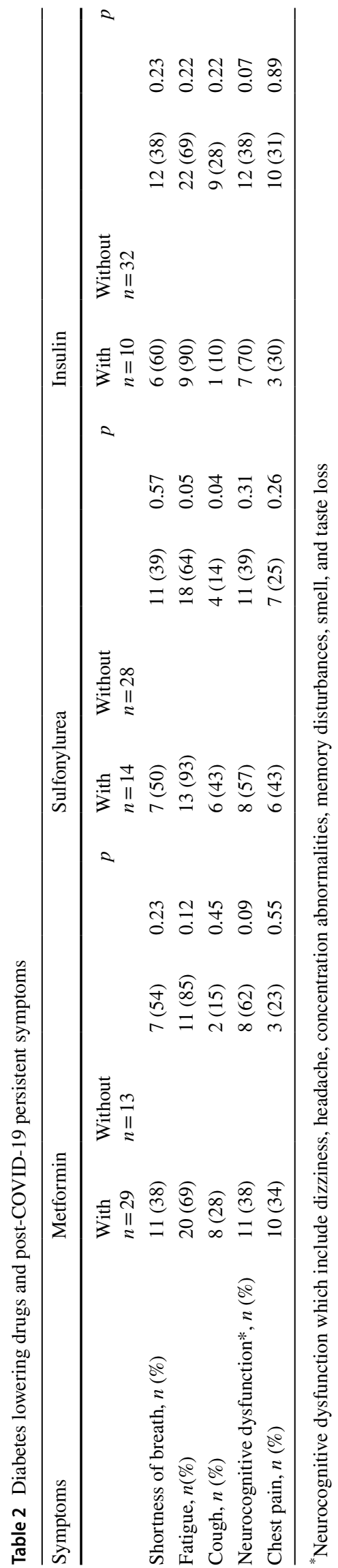

been suggested as potential mechanisms for long-term health consequences following acute viral infection [2, 4].

Based on recently available data, the coexistence of comorbid conditions, including older age and obesity, among patients with DM and COVID-19 infection, would predispose patients to adverse clinical outcomes and persistent symptoms [2, 3]. It has been suggested that COVID-19 infection may exhibit shared pathologies with DM and other comorbidities via immune dysfunction, chronic low-grade inflammation, high prevalence of preexisting comorbidities, and endothelial dysfunction [2].

According to available data, results related to DM lowering drugs and COVID-19 severity and prognosis have been inconsistent among clinical studies. Ethnic disparity, access to medical care, study design, number of enrolled patients, and frequency of adverse events in some studies populations may have influenced the inconsistent results of the literature [5].

The present study has several limitations. It is singlecenter study with a relatively small size population. This limitation did not allow us to eliminate the potential impacts of other confounders such as hypertension, older age, and obesity. Data regarding DM severity or duration before COVID-19 were not reported. Although symptoms were collected by a well-trained physician, we did not assess the severity of symptoms or perform quality of life or cognitive function assessments. There could be a possibility of selection bias as patients who attended the outpatient clinic were more likely to be more symptomatic.

Further studies are required to assess the pathogenesis and prognosis of long-term persistent symptoms following COVID-19 infection in patients with DM.

In conclusion, long-term persistent symptoms of COVID19 infection are common among patients with DM. Fatigue, neurocognitive dysfunction, shortness of breath, and chest pain are the most common symptoms among patients with DM.

\section{Declarations}

Conflict of interest The authors declare no competing interests.

\section{References}

1. Graham EL, Clark JR, Orban ZS, Lim PH, Szymanski AL, Taylor $\mathrm{C}$, et al. Persistent neurologic symptoms and cognitive dysfunction in non-hospitalized Covid-19 "long haulers." Ann Clin Transl Neurol. 2021;8(5):1073-85.

2. Feldman EL, Savelieff MG, Hayek SS, Pennathur S, Kretzler M, Pop-Busui R. COVID-19 and diabetes: a collision and collusion of two diseases. Diabetes. 2020;69(12):2549-65. 
3. Logue JK, Franko NM, McCulloch DJ, McDonald D, Magedson A, Wolf CR, et al. Sequelae in adults at 6 months after COVID-19 infection. JAMA Netw Open. 2021;4(2):e210830.

4. Huang C, Huang L, Wang Y, Li X, Ren L, Gu X, et al. 6-month consequences of COVID-19 in patients discharged from hospital: a cohort study. Lancet. 2021;397(10270):220-32.

5. Nafakhi H, Alareedh M, Al-Buthabhak K, Shaghee F, Nafakhi A, Kasim S. Predictors of adverse in-hospital outcome and recovery in patients with diabetes mellitus and COVID-19 pneumonia in Iraq. Diabetes Metab Syndr. 2021;15(1):33-8.

Publisher's note Springer Nature remains neutral with regard to jurisdictional claims in published maps and institutional affiliations. 